https://helda.helsinki.fi

\title{
Strain-Stiffening of Agarose Gels
}

\section{Bertula, Kia}

2019-06

Bertula , K, Martikainen , L , Munne , P , Hietala , S, Klefström , J , Ikkala , O \& Nonappa ,

D 2019 , ' Strain-Stiffening of Agarose Gels ' , Acs macro letters , vol. 8 , no. 6 , pp. 670-675 . https://doi.org/10.1021/acsmacrolett.9b00258

http://hdl.handle.net/10138/304522

https://doi.org/10.1021/acsmacrolett.9b00258

cc_by

publishedVersion

Downloaded from Helda, University of Helsinki institutional repository.

This is an electronic reprint of the original article.

This reprint may differ from the original in pagination and typographic detail.

Please cite the original version. 


\section{Strain-Stiffening of Agarose Gels}

Kia Bertula, ${ }^{\dagger, \ddagger}$ Lahja Martikainen, ${ }^{*}, \dagger,+$ Pauliina Munne, $"$ Sami Hietala, ${ }^{\perp \odot}$ Juha Klefström, Olli Ikkala, $*, \dagger, \S$ and Nonappa $*, \dagger, \S^{\circ}$

${ }^{\dagger}$ Department of Applied Physics, Molecular Materials Group, Aalto University School of Science, P.O. Box 15100, FI-00076, Espoo, Finland

${ }^{\S}$ Department of Bioproducts and Biosystems, Aalto University School of Chemical Engineering, P.O. Box 15100, FI-00076, Espoo, Finland

"Research Programs Unit/Translational Cancer Medicine Program and HiLIFE, University of Helsinki, P.O. Box 63, FI-00014, Helsinki, Finland

${ }^{\perp}$ Department of Chemistry, University of Helsinki, P.O. Box 55, FI-00014, HU, Helsinki, Finland

\section{Supporting Information}

ABSTRACT: Strain-stiffening is one of the characteristic properties of biological hydrogels and extracellular matrices, where the stiffness increases upon increased deformation. Whereas strain-stiffening is ubiquitous in protein-based materials, it has been less observed for polysaccharide and synthetic polymer gels. Here we show that agarose, that is, a common linear polysaccharide, forms helical fibrillar bundles upon cooling from aqueous solution. The hydrogels with these semiflexible fibrils show pronounced strain-stiffening. However, to reveal strain-stiffening, suppressing wall slippage turned as untrivial. Upon exploring different sample preparation techniques and rheological architectures, the cross-hatched parallel plate geometries and in situ gelation in the rheometer
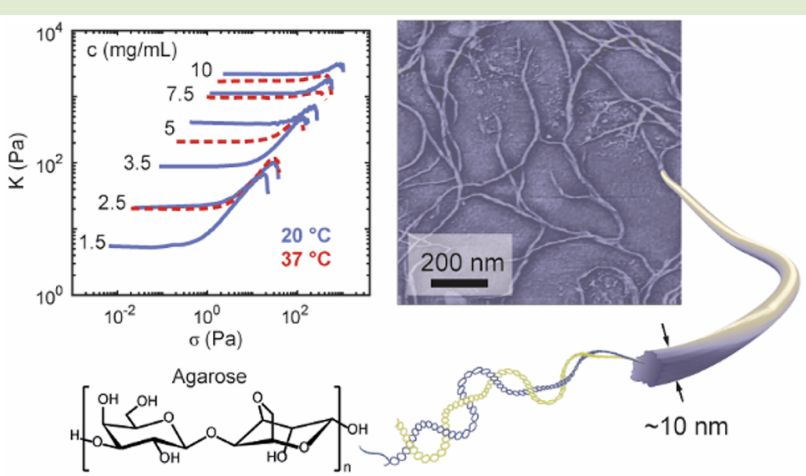
successfully prevented the slippage and resolved the strain-stiffening behavior. Combining with microscopy, we conclude that strain-stiffening is due to the semiflexible nature of the agarose fibrils and their geometrical connectivity, which is below the central-force isostatic critical connectivity. The biocompatibility and the observed strain-stiffening suggest the potential of agarose hydrogels in biomedical applications.

$\mathrm{H}$ ydrogels derived from biopolymers typically exhibit strain-stiffening, manifested as a sudden increase of the storage modulus under strain or stress. ${ }^{1}$ It is characteristically observed for hydrogels derived from biological tissues, such as collagen, neurofilaments, fibrin, and actin. ${ }^{1}$ Strain-stiffening is expected to be essential for the cell differentiation as well as for the integrity of tissues and the adaptation of extracellular matrices under mechanical deformation..$^{1-3}$ The nonlinear elasticity originates from the physical and chemical interactions of the fibrillar networks. Recently it has also been observed that gels derived from certain polysaccharides like alginate, ${ }^{4}$ methylcellulose, ${ }^{5,6}$ and pectin ${ }^{7,8}$ or even synthetic gels, ${ }^{9-11}$ can display strain-stiffening. Understanding and reliable characterization of strain-stiffening is relevant for many aspects of epithelial biology, stem cell culture, and tissue engineering applications. ${ }^{2}$

The strain-stiffening of a semiflexible fibrillar network depends on the interplay of the persistence length $l_{p}$, the contour length $L_{\mathcal{c}}$ and the topology of the connectivity (Figure 1A). The persistence length signifies the length where the thermal energy $k_{\mathrm{B}} T$ suffices to suppress the directional correlations of the fibril $l_{\mathrm{p}}=\kappa / k_{\mathrm{B}} T$, where $\kappa$ is the bending modulus. In semiflexible fibrils, the persistence length is comparable to the contour length $l_{\mathrm{p}} \approx L_{\mathrm{c}}$. Therefore, the bending rigidity prevents the entropic tendency of fibril to create loops or random coils, yet allowing some flexibility.

The wormlike chain model describes semiflexible fibrils, having finite bending stiffness. At small length scales $\left(<l_{\mathrm{p}}\right)$, their bending can be described by homogeneous rods in classical beam theory. ${ }^{12,13}$ A topological explanation of strainstiffening is then the low connectivity of a network (Figure 1A). ${ }^{14}$ Therein, the so-called central-force isostatic point (CFIP $)^{15-17}$ describes the critical connectivity as $z_{\text {CFIP }}=2 d(d$ $=$ dimensionality). Accordingly, if the average number of fibrils connecting in a node is above $z_{\text {CFIP }}$, the network is stiff, and below this threshold the network is floppy. For rigid and semiflexible polymer networks, below the $z_{\text {CFIP }}$ the main deformation mechanism is bending, whereas above this threshold it is stretching. ${ }^{18}$ Moreover, a network consisting of purely linear springs ${ }^{19}$ can show strain-stiffening if the

Received: April 8, 2019

Accepted: May 9, 2019

Published: May 23, 2019 
A)

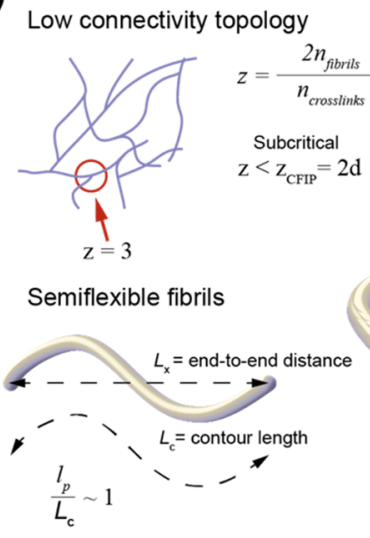

B)

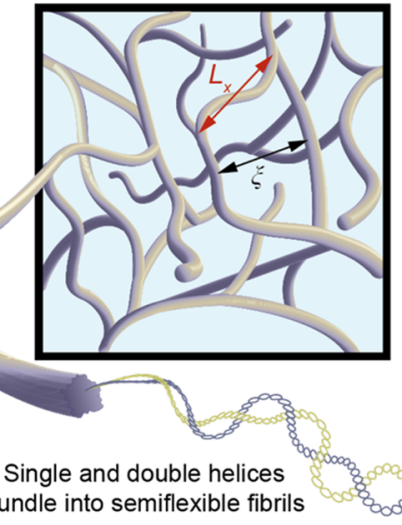

C)

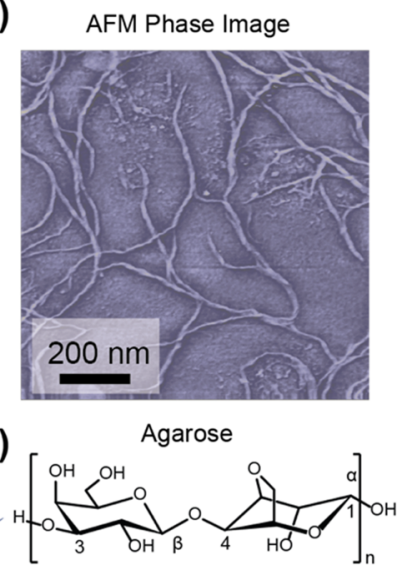

Figure 1. (A) The strain-stiffening of a network originates from the low connectivity $z<6$ (in $3 \mathrm{D}$ ) and the semiflexible nature of the fibrils with $l_{p} \approx$ $L_{c}$. (B) Schematic representation of gel networks. $L_{x}$ (red arrow) indicates the length between connecting points, i.e., the end-to-end distance of the fibrils in the network, and $\xi$ (black arrow) shows the mesh size. (C) Atomic force microscopy phase image of an agarose fibrillar network showing the helically twisted bundles. (D) Chemical structure showing the repeat units of agarose.

connectivity is below the CFIP ( $<6$ in $3 \mathrm{D})$. The fibrils might align upon shearing, which further increases the stiffness. ${ }^{20-22}$

Agarose $^{23}$ is a linear polysaccharide extracted from seaweed. It is one of the main components of agar, a mixture of agarose and agaropectin. It consists of alternating D-galactose and 3,6anhydro-L-galactopyranose units linked by $\alpha-(1 \rightarrow 3)$ and $\beta$-(1 $\rightarrow 4$ ) glycosidic bonds (Figure 1D). Agarose forms gels when dispersed in water or mixtures of water and selected cosolvents. $^{24}$ The gelation depends on the agarose concentration and molecular weight. Apart from their extensive use in the food industry, electrophoresis for biopolymer purification, agarose gels have been studied for cell culturing and tissue engineering due to the biocompatibility. ${ }^{25}$ At high temperatures, agarose forms random coils in aqueous solutions. Upon cooling, it forms both single- and double-helices, which bundle and assemble into fibrillar networks. ${ }^{26}$ Some studies hint that agar gels $^{27,28}$ and agarose gels ${ }^{29}$ could undergo strain-stiffening, but direct rheological measurements have generally failed to display this property.

Here, we describe the strain-stiffening of agarose hydrogels using oscillatory rheology. We will describe the crucial role of the sample preparation and rheological measurement setup to prevent the wall slippage, which can hinder the observation of strain-stiffening. Furthermore, combined atomic force microscopy (AFM), cryogenic transmission electron microscopy (cryo-TEM), and scanning electron microscopy (SEM) reveals the helical bundling to semiflexible fibrils and their connectivity in the nodal points.

We selected mainly low-gelling temperature $\left(T_{\text {gel }}=25^{\circ} \mathrm{C}\right)$ agarose with number-average molar mass $M_{\mathrm{n}} \approx 109000 \mathrm{~g} / \mathrm{mol}$, weight-average molar mass $M_{\mathrm{w}} \approx 139000 \mathrm{~g} / \mathrm{mol}$, and polydispersity index of 1.28 (Figure S2). Hydrogels were prepared by first dispersing the agarose powder in phosphatebuffered saline (PBS, relevant for biological applications) to obtain a clear solution upon heating, followed by cooling to the room temperature. The rheological measurements were conducted at $20{ }^{\circ} \mathrm{C}$ and $37{ }^{\circ} \mathrm{C}$ considering the prospective biomedical applications. Data were acquired in triplicate and reported as average unless otherwise stated.

Sample preparation and different geometries influence the results in the nonlinear viscoelastic regime. ${ }^{30}$ Therefore, three different geometries were used for rheology: (i) a smooth parallel plate, (ii) cross-hatched parallel plate, and (iii) cone and plate (smooth). We used $5.0 \mathrm{mg} / \mathrm{mL}$ aq. (PBS) agarose gels using two different sample preparation methods, namely, ex situ and in situ methods. For the ex situ approach, preprepared disc-shape (molded) samples were used either with a smooth or a cross-hatched parallel plate geometry. For the in situ gelation, the hot sols were allowed to gel on the rheometer and measured with a normal force control setup due to the volume contraction of the gels during the gelling. ${ }^{31}$ The normal force was kept as a constant $\left(F_{\mathrm{N}}=0.0 \pm 0.1 \mathrm{~N}\right)$, allowing the gap to vary according to the volume contraction during the gelation. Besides, a cone and plate geometry was also used for the rheological measurements of in situ gelation. More detailed sample preparation methods and geometries are described in the Supporting Information (Figure S1, Table S1).

To compare the data extracted using different geometries, the storage moduli $\left(G^{\prime}\right)$ from the strain amplitude sweeps were normalized with the corresponding linear viscoelastic moduli $G_{\text {LVR }}^{\prime}$ (Figure 2A). Among all cases, strain-stiffening was observed only with in situ samples using the cross-hatched geometry. In situ prepared samples with a smooth parallel plate or cone and plate geometry did not consistently show strainstiffening. The ex situ samples indicated strain-softening behavior, irrespective of the geometry. In conclusion, the geometry and sample preparation methods control the wall slip. This is relevant, as the wall slip results in an incomplete force transfer to the sample, leading to erroneous and unreliable data. Wall slip can be revealed from the raw waveform data or normalized Lissajous figures. ${ }^{30,32}$ This is not, however, unambiguous, because yielding has a similar effect on the stress waveform. ${ }^{33,34}$ Slippage can also be observed in the maximum of loss modulus $\left(G^{\prime \prime}\right)$ upon increasing strain/stress. At this point the stress waveform deviates from a sinusoidal response with the two "additional peaks". ${ }^{35}$ Our rheological studies revealed a typical stress waveform (Figure S4B) and a distinct strain-stiffening Lissajous figure for in situ prepared hydrogel sample with cross-hatched geometry (Figure S4E, see SI for a detailed analysis). Further, for in situ samples with the cross-hatched geometry, the strain-stiffening was retained for the gaps between $250 \mu \mathrm{m}$ and $1.0 \mathrm{~mm}$ (Figure S5). 


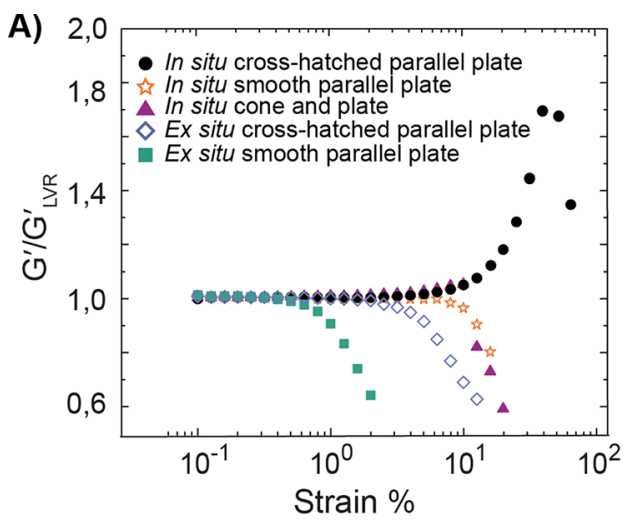

B)

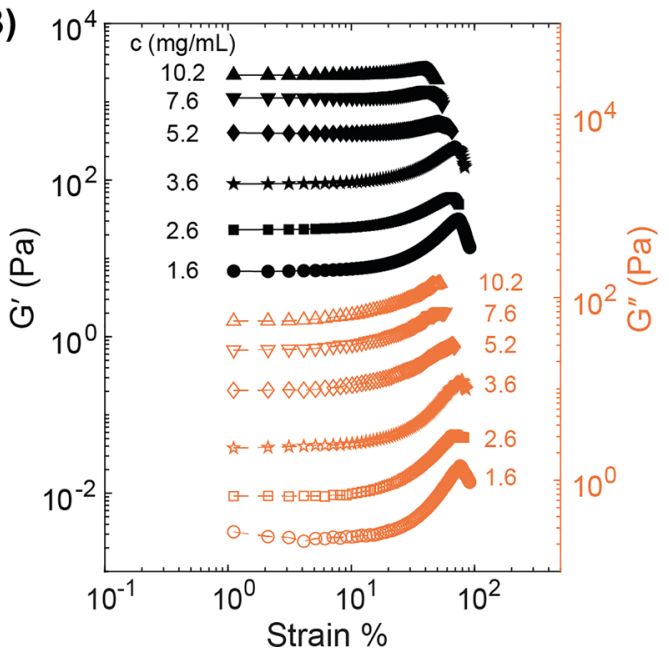

Figure 2. Oscillatory rheology of the agarose gels in PBS buffer at 20 ${ }^{\circ}$ C. (A) Strain sweeps with normalized $G^{\prime}$ for different sample preparation methods and geometries showing strain-stiffening only using an in situ sample preparation method with a cross-hatched parallel plate geometry, leading to reduced wall slip. (B) Representative strain amplitude sweeps of agarose gels using in situ prepared cross-hatched parallel plate geometries with different concentrations in aq. PBS showing the strain-stiffening response $\left(G^{\prime}\right.$ = black filled symbols, $G^{\prime \prime}=$ orange open symbols).

After having screened the proper conditions to resolve the strain-stiffening, agarose gels were systematically studied under in situ conditions with the cross-hatched parallel plate geometry. Accordingly, hydrogels with six different aq. concentrations $(1.6,2.6,3.6,5.2,7.6$, and $10.2 \mathrm{mg} / \mathrm{mL})$ at $20{ }^{\circ} \mathrm{C}$ and four different concentrations $(2.6,5.1,7.7,10.2 \mathrm{mg} /$ $\mathrm{mL}$ ) at $37{ }^{\circ} \mathrm{C}$ in PBS were studied (see Figures $2 \mathrm{~B}$ and S6S12). Strain-stiffening is observed as an increase of storage modulus $\left(G^{\prime}\right)$ above a threshold strain in all concentrations and also in the loss modulus $\left(G^{\prime \prime}\right)$. A further increase in the amplitude strain results in a drop in the moduli either due to initiation of wall-slippage or due to gel breaking or a combination of both. Strain-stiffening was also confirmed for agarose gels prepared in pure water (Figure S13). Also, hydrogels derived from agaroses of different gelling temperature $\left(T_{\text {gel }}=36{ }^{\circ} \mathrm{C}\right.$ and $\left.T_{\text {gel }}=8-17{ }^{\circ} \mathrm{C}\right)$ showed strainstiffening (Figure S14). Therefore, we suggest that strainstiffening is universal for agarose gels.

The network structures of the agarose gels were analyzed with different microscopies (Figures 3, S7, S17, and S18). Cryo-TEM images of the freshly prepared agarose gels revealed
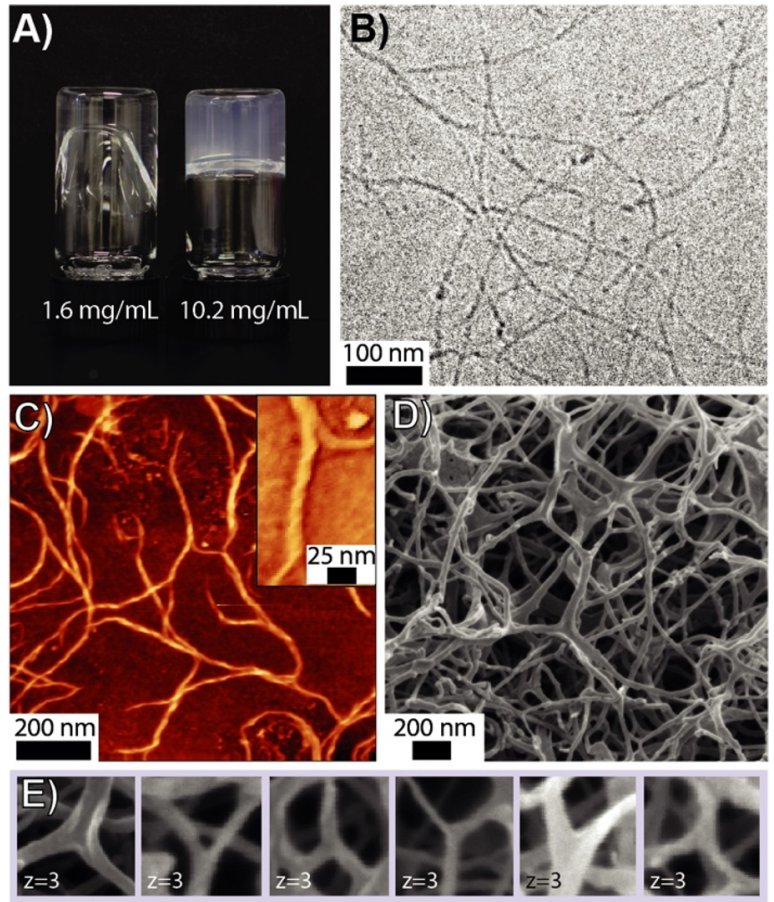

$1.6 \mathrm{mg} / \mathrm{mL} \quad 2.5 \mathrm{mg} / \mathrm{mL} \quad 3.5 \mathrm{mg} / \mathrm{mL} \quad 5.1 \mathrm{mg} / \mathrm{mL} \quad 7.6 \mathrm{mg} / \mathrm{mL} \quad 10.2 \mathrm{mg} / \mathrm{mL}$

Figure 3. Network structure of the agarose gels. (A) Photograph of 1.6 and $10.2 \mathrm{mg} / \mathrm{mL}$ agarose aqueous gels in water. (B) Cryo-TEM image of $0.83 \mathrm{mg} / \mathrm{mL}$ gel. (C) AFM height image of $5.11 \mathrm{mg} / \mathrm{mL}$ gel. The inset shows the AFM phase image of a helically twisted bundle. (D) SEM image of $1.6 \mathrm{mg} / \mathrm{mL}$ gel. (E) Connectivity $(z \approx 3)$ obtained from SEM images of different concentrations.

long shape-persistent fibrils of a diameter of $\sim 10 \mathrm{~nm}$ (Figures $3 \mathrm{~B}$ and S18). AFM images revealed that the agarose fibrils have helical twisting (Figures 1B, 3C, and S17) and persistence length, $l_{\mathrm{p}}$, of $1278 \pm 449 \mathrm{~nm}$ estimated via Easyworm software $^{36}$ (Figure S20). The overall morphology was analyzed using SEM images of aerogels prepared from agarose gels from different concentrations by freezing in liquid propane followed by lyophilization (Figures 3D, S7, and S19). The liquid propane freezing step allows a quick cooling in comparison to liquid nitrogen freezing due to absence of the Leidenfrost effect. $^{37}$ This reduces the tendency for fibril aggregation. The fibril diameter (6.3-19.7 nm, see Table S3 and Figures S15S18) and the network structures were qualitatively similar in this concentration range. All agarose gels appear to contain a range of different mesh sizes 10-1000 nm (see Figures S7, S18, and S19). Therefore, a single mesh size (pore-size) value cannot be given. Moreover, an unambiguous characterization of the network pore-size in agarose hydrogels using AFM or SEM micrograph analysis is challenging due to the possible artifacts arising from the specimen preparation. Therefore, previously the determination of the fibril radii and pore-sizes have been carried out using multiple complementary techniques, including NMR spectroscopy, electrophoresis, light scattering, and turbidity measurements. ${ }^{38}$ It has been shown that the lateral dimension of the fibrils is independent of the agarose concentration. On the other hand, the pore-size depends on agarose type, concentration, and gelation kinetics, leading to values ranging from 80 to $500 \mathrm{~nm} .{ }^{38}$ Our results on the fiber diameter and pore-size are consistent with the earlier literature reports. The agarose gels contain fibril rich (dense network) and fibril poor (sparse network) areas, which are a 
result of the gelling kinetics at this concentration range. ${ }^{39}$ Yet, $L_{\mathcal{c}}, l_{\mathrm{p}}$, and $\xi$ are at the same length scale (also seen as slightly bent fibrils in micrographs), indicating that the network consists of semiflexible fibrils. Connectivity $z$ at the nodal points for different concentrations was analyzed from SEM images. Note that the exact values for $z$ are challenging to estimate from SEM as the sample preparation and drying can affect the final network structure and as the fibrils overlap. Still, $z \approx 3$ in all concentrations, with a few exceptions, which is definitely below the central-force isostatic point $z_{\text {CFIP }}=6$ (Figures 3E and S19).

To gain more insight, the differential modulus $K=\partial \sigma / \partial \gamma$ (Figure 4A, inset) is calculated from the strain sweep data at 20 ${ }^{\circ} \mathrm{C}$ (Figure 2B) and $37{ }^{\circ} \mathrm{C}$ (Figure S9D), plotted as a function of the shear stress $\sigma$ in Figure 4A, see also Figure S10A as a function of strain. Near the zero shear stress or strain, $K$ is close to the zero stress storage modulus $G_{0}$. As the
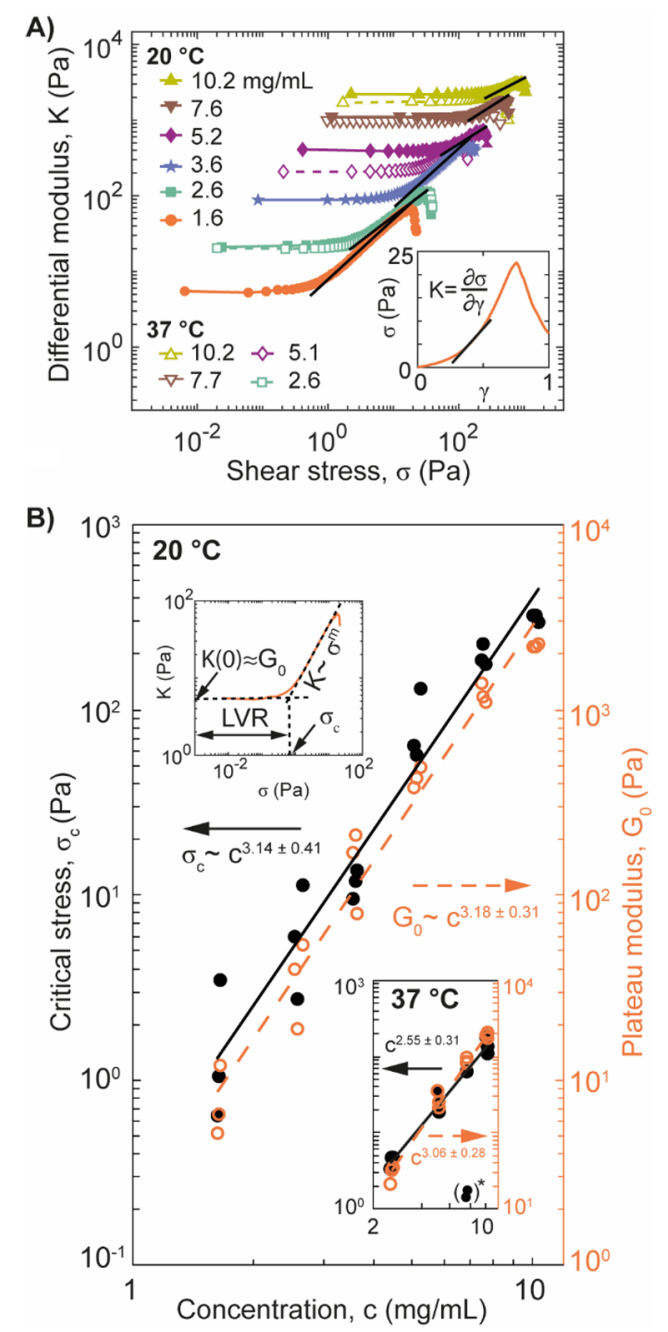

Figure 4. Strain-stiffening of the agarose gels at $20{ }^{\circ} \mathrm{C}$ (filled symbols and solid lines) and $37^{\circ} \mathrm{C}$ (open symbols and dashed lines). (A) Differential modulus $K$ as a function of shear stress $\sigma$ at $20^{\circ} \mathrm{C}$ (six different concentrations) and $37^{\circ} \mathrm{C}$ (four different concentrations). Here we show only representative measurements per each concentration-temperature combination. (B) Critical stress and the plateau modulus as a function of agarose concentration (main) at 20 ${ }^{\circ} \mathrm{C}$ and (lower inset) at $37{ }^{\circ} \mathrm{C}$. ${ }^{*}$ Two specimens at $7.7 \mathrm{mg} / \mathrm{mL}$ showed very little stiffening because of the slippage, excluded from the $\sigma_{\mathrm{c}}$ fit. The upper inset shows how critical stress is obtained. concentration increases, the slopes of the strain-stiffening regions decrease; however, it is likely that the decrease of the slope comes at least partly from the slippage, as the slippage is hard to prevent completely, especially at high concentrations with the current setup. Moreover, agarose gels release water between the gel and rheometer plates with time (syneresis), ${ }^{23}$ which further promotes the slippage. Yet the curves collapse into the same threshold point when data is normalized (Figure S10C). Therefore, the strain-stiffening is stress controlled following power law dependency $K \sim \sigma^{0.80}$.

Biopolymer networks like actin, neurofilaments, fibrin, and synthetic polyisocyanopeptide hydrogels show universal scaling of $K \sim \sigma^{3 / 2}$ at the strain-stiffening regime, which originates from the force-extension relation of individual fibrils. ${ }^{1}$ This universal behavior holds only if the main deformation mechanism is entropic and the fibrils are inextensible. ${ }^{12}$ Polymers inherently have some purely enthalpic extension as opposed to entropic, that might affect the strain-stiffening behavior at high stress, resulting in a deviation from the universal behavior. Here, the lower exponent (0.80) observed for agarose hydrogels indicate that the networks can be considered as athermal showing enthalpic elasticity. However, we cannot exclude the possible slippage, which might as well result in lower scaling. A comparison of effective entropic and enthalpic spring constants show that the dominant mechanical response to stretching is enthalpic only if $L_{x}{ }^{3}<r^{2} l_{\mathrm{p}}$, where $L_{x}$ is the distance between the two connecting points and $r$ is the radius of the fibril. ${ }^{12}$ Here, $r=3.15-9.85 \mathrm{~nm}$ and $l_{\mathrm{p}}=1278 \pm$ $449 \mathrm{~nm}$ (Figure S20). If $L_{x}$ is less than $20-55 \mathrm{~nm}$, the primary mechanical response is expected to be enthalpic. In agarose hydrogels, the mesh size varies from tens of nanometers up to several hundred nanometers or micrometer scale (see Figures 3, S7, S18, and S19), suggesting that the response of sparse areas is entropic, while denser parts mainly show enthalpic elasticity.

Figure 4B shows the scaling behavior of the critical stress $\sigma_{\mathrm{c}}$ and zero stress storage modulus $G_{0}$. In this work, the critical stress refers to the stress at which the stiffening sets in. Here the zero-stress storage modulus is presented as a plateau modulus $G_{0}$, which is calculated as an average storage modulus from the frequency sweeps with $1 \%$ strain amplitude between $0.1194-4.755 \mathrm{rad} / \mathrm{s}$ after the gelling time sweep step. Both $G_{0}$ and $\sigma_{\mathrm{c}}$ follow roughly the same power law relationship as a function of concentration at $20{ }^{\circ} \mathrm{C}: G_{0} \sim c^{3.18 \pm 0.31}$ and $\sigma_{\mathrm{c}} \sim$ $c^{3.14 \pm 0.41}$, showing the standard errors. Though it is surprising to get a similar scaling with plateau modulus $G_{0}$ and critical stress $\sigma_{\mathcal{c}}$ such observations have also been made earlier. For polyisocyanopeptide hydrogel networks, the $G_{0}$ and $\sigma_{\mathrm{c}}$ had the same scaling (in their case, $\sim c^{2}$ ). ${ }^{40}$ Simulations for an affine thermal model for cross-linked networks have suggested that the plateau modulus $G_{0}$ and critical stress $\sigma_{\mathrm{c}}$ scale almost with same exponents $\left(G_{0} \sim c^{11 / 5}\right.$ and $\left.\sigma_{\mathrm{c}} \sim c^{9 / 5}\right){ }^{12}$ Computational models of subisostatic athermal fiber networks suggest that the linear dependency between the modulus and the critical stress follows naturally from the hypothesis of normal stress-induced stiffening. ${ }^{41}$ At $37{ }^{\circ} \mathrm{C}$ the present scaling relations slightly change to $G_{0} \sim c^{3.06 \pm 0.28}$ and $\sigma_{\mathrm{c}} \sim c^{2.55 \pm 0.31}$. There, the scaling exponent is slightly lower than observed for gels at $20{ }^{\circ} \mathrm{C}$. However, taking into account the standard error and the fact that the gels at higher concentrations $(c>5 \mathrm{mg} / \mathrm{mL})$ tend to slip more easily at $37{ }^{\circ} \mathrm{C}$ (Figures S11 and S12), the scaling is more likely to be the same or at least close to 3 , like those obtained for gels at $20{ }^{\circ} \mathrm{C}$. Our results are consistent with a 
recent report, ${ }^{42}$ where the storage modulus of agarose gels follows the power law $G^{\prime} \sim c^{3}$ for $0.1-0.35$ wt \%. Such scaling exponent is larger than that observed earlier for agarose ${ }^{43}$ and those of the biopolymer gels showing the scaling exponent 2$2.5 .^{44,45}$ Stronger scaling with concentration could hint that the connectivity of the network might increase slightly upon increasing the agarose concentration. ${ }^{45}$ However, here we do not detect differences in connectivity (Figure 3E).

If the connectivity increases as a function of concentration, it should decrease the critical strain $\gamma_{c}$ for the onset of strainstiffening. ${ }^{17,46}$ Here $\gamma_{c}$ increases gradually upon concentration at $20^{\circ} \mathrm{C}$, and a slight rise is also seen at $37^{\circ} \mathrm{C}$ (Figure S10B). This suggests that the elasticity of agarose networks could be covered with athermal models of disordered fiber networks showing enthalpic elasticity. ${ }^{41}$ To address in future whether the agarose networks are truly in the regime of entropic or enthalpic elasticity, the anisotropy of the network structure and nonaffinity of the deformation should be inspected, for example, by studying carefully the normal stresses ${ }^{47}$ or using visualizing technique coupled to rheometer. ${ }^{48}$

In conclusion, significant strain-stiffening was observed for agarose gels taken that wall slippage in rheometry could be minimized by in situ gelation within cross-hatched parallel plate geometry. Moreover, the sample preparation turned critical: strain-stiffening was not observed if (i) ex situ readymade gels were inserted to smooth or cross-hatched parallel plate geometries, (ii) if the gel is prepared in situ with smooth parallel plate or cone and plate geometries, all of such cases promoting slippage. Strain-stiffening was obtained using various agarose concentrations (between 1.6 to $10.2 \mathrm{mg}$ / $\mathrm{mL})$, two different temperature $\left(20{ }^{\circ} \mathrm{C}\right.$ and $\left.37{ }^{\circ} \mathrm{C}\right)$ and two solvents ( $1 \times \mathrm{PBS}$ and water) with three different commercial agaroses. Therefore, the strain-stiffening is generic for agarose gels. We show that the strain-stiffening is strain induced, having a gradual dependency on agarose concentration. The network structure dictates the critical strain. The fibril diameter, fibril persistence length, and network connectivity remain practically the same between the concentrations 1.5$10 \mathrm{mg} / \mathrm{mL}$. An increase in agarose concentration results in a slight decrease in average mesh size and raise in the bending modulus of fibril segments between the cross-linking points, which causes the observed increase of critical strain. ${ }^{41}$ In contrast, the critical stress $\sigma_{\mathrm{c}}$, where the strain-stiffening starts, depends directly on the original storage modulus $G_{0}$ of the gel and the strain-stiffening regime is stress controlled following power law $K \sim \sigma^{0.80}$. Both $G_{0}$ and $\sigma_{\mathrm{c}}$ follow roughly the same power law as a function of concentration, that is, $G_{0} \sim c^{3.18}$ and $\sigma_{\mathrm{c}} \sim c^{3.14}$ at $20{ }^{\circ} \mathrm{C} G_{0} \sim c^{3.06}$ and $\sigma_{\mathrm{c}} \sim c^{2.55}$ at $37{ }^{\circ} \mathrm{C}$, respectively. Our results imply that the agarose gels are athermal fibril networks and the strain-stiffening is covered by semiflexible nature of the agarose fibrils and due to the low connectivity $(z \approx 3)$, which is below the central-force isostatic point. This work suggests to explore new application potential for agarose gels and refreshed rheological explorations of other polysaccharide gels to reveal strain-stiffening.

\section{ASSOCIATED CONTENT}

\section{S Supporting Information}

The Supporting Information is available free of charge on the ACS Publications website at DOI: 10.1021/acsmacrolett.9b00258.
Materials and methods, size-exclusion chromatography, rheological measurements and analysis, sample preparation methods, additional SEM, AFM images, and cryoTEM images, analysis of fiber diameter, connectivity and persistence length (PDF)

\section{AUTHOR INFORMATION}

\section{Corresponding Authors}

*E-mail lahja.martikainen@aalto.fi.

*E-mail olli.ikkala@aalto.fi.

*E-mail nonappa@aalto.fi.

ORCID

Sami Hietala: 0000-0003-1448-1813

Olli Ikkala: 0000-0002-0470-1889

Nonappa: 0000-0002-6804-4128

\section{Author Contributions}

† These authors contributed equally. All authors have given approval to the final version of the manuscript.

\section{Funding}

Academy of Finland Centre of Excellence (HYBER 20142019), Business Finland (Grant Nos. 544/31/2015 and 2489/ 31/2017), and ERC-Advanced Grant (DRIVEN) are acknowledged. L.M. acknowledges Jenny and Antti Wihuri Foundation. L.M. and K.B. acknowledge Walter Ahlström Foundation. K.B. acknowledges Tekniikan edistämissäätiö.

Notes

The authors declare no competing financial interest.

\section{ACKNOWLEDGMENTS}

This work made use of the Aalto University Nanomicroscopy Center (Aalto-NMC) premises. The authors acknowledge Leena Pitkänen from Department of Bioproducts and Biosystems at Aalto University for the SEC measurements.

\section{REFERENCES}

(1) Storm, C.; Pastore, J. J.; MacKintosh, F. C.; Lubensky, T. C.; Janmey, P. A. Nonlinear Elasticity in Biological Gels. Nature 2005, 435, 191-194.

(2) Das, R. K.; Gocheva, V.; Hammink, R.; Zouani, O. F.; Rowan, A. E. Stress-Stiffening-Mediated Stem-Cell Commitment Switch in Soft Responsive Hydrogels. Nat. Mater. 2016, 15, 318-325.

(3) Wen, Q.; Janmey, P. A. Effects of Nonlinearity on Cell-ECM Interactions. Exp. Cell Res. 2013, 319, 2481-2489.

(4) Hashemnejad, S. M.; Kundu, S. Strain Stiffening and Negative Normal Stress in Alginate Hydrogels. J. Polym. Sci., Part B: Polym. Phys. 2016, 54, 1767-1775.

(5) McKee, J. R.; Hietala, S.; Seitsonen, J.; Laine, J.; Kontturi, E.; Ikkala, O. Thermoresponsive Nanocellulose Hydrogels with Tunable Mechanical Properties. ACS Macro Lett. 2014, 3, 266-270.

(6) McAllister, J. W.; Lott, J. R.; Schmidt, P. W.; Sammler, R. L.; Bates, F. S.; Lodge, T. P. Linear and Nonlinear Rheological Behavior of Fibrillar Methylcellulose Hydrogels. ACS Macro Lett. 2015, 4, 538542 .

(7) Schuster, E.; Lundin, L.; Williams, M. A. K. Investigating the Relationship between Network Mechanics and Single-Chain Extension Using Biomimetic Polysaccharide Gels. Macromolecules 2012, 45, 4863-4869.

(8) Vincent, R. R. R.; Mansel, B. W.; Kramer, A.; Kroy, K.; Williams, M. A. K. Micro-Rheological Behaviour and Nonlinear Rheology of Networks Assembled from Polysaccharides from the Plant Cell Wall. New J. Phys. 2013, 15, 1-21.

(9) Tung, S.-H.; Raghavan, S. R. Strain-Stiffening Response in Transient Networks Formed by Reverse Wormlike Micelles. Langmuir 2008, 24, 8405-8408. 
(10) Fernandez-Castano Romera, M.; Lafleur, R. P. M.; Guibert, C.; Voets, I. K.; Storm, C.; Sijbesma, R. P. Strain Stiffening Hydrogels through Self-Assembly and Covalent Fixation of Semi-Flexible Fibers. Angew. Chem., Int. Ed. 2017, 56, 8771-8775.

(11) Kouwer, P. H. J.; Koepf, M.; Le Sage, V. A.A.; Jaspers, M.; van Buul, A. M.; Eksteen-Akeroyd, Z. H.; Woltinge, T.; Schwartz, E.; Kitto, H. J.; Hoogenboom, R.; Picken, S. J.; Nolte, R. J. M.; Mendes, E.; Rowan, A. E. Responsive Biomimetic Networks from Polyisocyanopeptide Hydrogels. Nature 2013, 493, 651-655.

(12) Broedersz, C. P.; MacKintosh, F. C. Modeling Semiflexible Polymer Networks. Rev. Mod. Phys. 2014, 86, 995-1036.

(13) Meng, F.; Terentjev, E. M. Theory of Semiflexible Filaments and Networks. Polymers (Basel, Switz.) 2017, 9, 1-28.

(14) Maxwell, J. C. On the Calculation of the Equilibrium and Stiffenss of Frames. Philos. Mag. 1864, 27, 294.

(15) Feng, J.; Levine, H.; Mao, X.; Sander, L. M. Nonlinear Elasticity of Disordered Fiber Networks. Soft Matter 2016, 12, 1419-1424.

(16) Broedersz, C. P.; Mao, X.; Lubensky, T. C.; MacKintosh, F. C. Criticality and Isostaticity in Fibre Networks. Nat. Phys. 2011, 7, 983-988.

(17) Sharma, A.; Licup, A. J.; Jansen, K. A.; Rens, R.; Sheinman, M.; Koenderink, G. H.; MacKintosh, F. C. Strain-Controlled Criticality Governs the Nonlinear Mechanics of Fibre Networks. Nat. Phys. 2016, 12, 584-588.

(18) Heussinger, C.; Schaefer, B.; Frey, E. Nonaffine Rubber Elasticity for Stiff Polymer Networks. Phys. Rev. E 2007, 76, 031906.

(19) Sheinman, M.; Broedersz, C. P.; MacKintosh, F. C. Nonlinear Effective-Medium Theory of Disordered Spring Networks. Phys. Rev. E 2012, 85, 1-16.

(20) Bouzid, M.; Del Gado, E. Network Topology in Soft Gels: Hardening and Softening Materials. Langmuir 2018, 34, 773-781.

(21) Feng, J.; Levine, H.; Mao, X.; Sander, L. M. Alignment and Nonlinear Elasticity in Biopolymer Gels. Phys. Rev. E 2015, 91, 042710 .

(22) Onck, P. R.; Koeman, T.; van Dillen, T.; van Der Giessen, E. Alternative Explanation of Stiffening in Cross-Linked Semiflexible Networks. Phys. Rev. Lett. 2005, 95, 178102.

(23) Rinaudo, M. Main Properties and Current Applications of Some Polysaccharides as Biomaterials. Polym. Int. 2008, 57, 397-430.

(24) Ramzi, M.; Rochas, C.; Guenet, J. M. Structure-Properties Relation for Agarose Thermoreversible Gels in Binary Solvents. Macromolecules 1998, 31, 6106-6111.

(25) Zarrintaj, P.; Manouchehri, S.; Ahmadi, Z.; Saeb, M. R.; Urbanska, A. M.; Kaplan, D. L.; Mozafari, M. Agarose-Based Biomaterials for Tissue Engineering. Carbohydr. Polym. 2018, 187, $66-84$.

(26) Rüther, A.; Forget, A.; Roy, A.; Carballo, C.; Mießmer, F.; Dukor, R. K.; Nafie, L. A.; Johannessen, C.; Shastri, V. P.; Ludeke, S. Unravelling a Direct Role for Polysaccharide b -Strands in the Higher Order Structure of Physical Hydrogels. Angew. Chem., Int. Ed. 2017, 56, 4603-4607.

(27) Altmann, N.; Cooper-White, J. J.; Dunstan, D. E.; Stokes, J. R. Strong through to Weak 'Sheared' Gels. J. Non-Newtonian Fluid Mech. 2004, 124, 129-136.

(28) Sousa, A. M. M.; Gonçalves, M. P. The Influence of Locust Bean Gum on Native and Alkali-Modified Agargels. Food Hydrocolloids 2015, 44, 461-470.

(29) Mao, B.; Bentaleb, A.; Louerat, F.; Divoux, T.; Snabre, P. HeatInduced Aging of Agar Solutions: Impact on the Structural and Mechanical Properties of Agar Gels. Food Hydrocolloids 2017, 64, 5969.

(30) Walter, B. L.; Pelteret, J. P.; Kaschta, J.; Schubert, D. W.; Steinmann, P. On the Wall Slip Phenomenon of Elastomers in Oscillatory Shear Measurements Using Parallel-Plate Rotational Rheometry: I. Detecting Wall Slip. Polym. Test. 2017, 61, 430-440.

(31) Mao, B.; Divoux, T.; Snabre, P. Normal Force Controlled Rheology Applied to Agar Gelation. J. Rheol. (Melville, NY, U. S.) 2016, 60, 473-489.
(32) Walter, B. L.; Pelteret, J. P.; Kaschta, J.; Schubert, D. W.; Steinmann, P. On the Wall Slip Phenomenon of Elastomers in Oscillatory Shear Measurements Using Parallel-Plate Rotational Rheometry: II. Influence of Experimental Conditions. Polym. Test. 2017, 61, 455-463.

(33) Yoshimura, A. S.; Prud'homme, R. K. Wall Slip Effects on Dynamic Oscillatory Measurements. J. Rheol. (Melville, NY, U. S.) 1988, 32, 575-584.

(34) Yoshimura, A. S.; Prud'homme, R. K. Response of an Elastic Bingham Fluid to Oscillatory Shear. Rheol. Acta 1987, 26, 428-436.

(35) Kang, H.; Wen, Q.; Janmey, P. A.; Tang, J. X.; Conti, E.; MacKintosh, F. C. Nonlinear Elasticity of Stiff Filament Networks: Strain Stiffening, Negative Normal Stress, and Filament Alignment in Fibrin Gels. J. Phys. Chem. B 2009, 113, 3799-3805.

(36) Lamour, G.; Kirkegaard, J. B.; Li, H.; Knowles, T. P. J.; Gsponer, J. Easyworm: An Open-Source Software Tool to Determine the Mechanical Properties of Worm-like Chains. Source Code Biol. Med. 2014, 9, 1-6.

(37) Korhonen, J. T.; Hiekkataipale, P.; Malm, J.; Karppinen, M.; Ikkala, O.; Ras, R. H. A. Inorganic Hollow Nanotube Aerogels by Atomic Layer Deposition onto Native Nanocellulose Templates. ACS Nano 2011, 5, 1967-1974.

(38) Aymard, P.; Martin, D. R.; Plucknett, K.; Foster, T. J.; Clark, A. H.; Norton, I. T. Influence of Thermal History on the Structural and Mechanical Properties of Agarose Gels. Biopolymers 2001, 59, 131144.

(39) San Biagio, P. L.; Bulone, D.; Emanuele, A.; Palma-Vittorelli, M. B.; Palma, M. U. Spontaneous Symmetry-Breaking Pathways: Time-Resolved Study of Agarose Gelation. Food Hydrocolloids 1996, 10, 91-97.

(40) Jaspers, M.; Dennison, M.; Mabesoone, M. F. J.; MacKintosh, F. C.; Rowan, A. E.; Kouwer, P. H. J. Ultra-Responsive Soft Matter from Strain-Stiffening Hydrogels. Nat. Commun. 2014, 5, 5808.

(41) Licup, A. J.; Sharma, A.; MacKintosh, F. C. Elastic Regimes of Subisostatic Athermal Fiber Networks. Phys. Rev. E: Stat. Phys., Plasmas, Fluids, Relat. Interdiscip. Top. 2016, 93, 012407.

(42) Le Goff, K. J.; Gaillard, C.; Helbert, W.; Garnier, C.; Aubry, T. Rheological Study of Reinforcement of Agarose Hydrogels by Cellulose Nanowhiskers. Carbohydr. Polym. 2015, 116, 117-123.

(43) Fujii, T.; Yano, T.; Kumagai, H.; Miyawaki, O. Scaling Analysis of the Concentration Dependence on Elasticity of Agarose Gel. Biosci., Biotechnol., Biochem. 2000, 64, 1618-1622.

(44) MacKintosh, F. C.; Käs, J.; Janmey, P. A. Elasticity of Semiflexible Biopolymer Networks. Phys. Rev. Lett. 1995, 75, 44254428.

(45) Lin, Y. C.; Yao, N. Y.; Broedersz, C. P.; Herrmann, H.; MacKintosh, F. C.; Weitz, D. A. Origins of Elasticity in Intermediate Filament Networks. Phys. Rev. Lett. 2010, 104, 058101.

(46) Jansen, K. A.; Licup, A. J.; Sharma, A.; Rens, R.; MacKintosh, F. C.; Koenderink, G. H. The Role of Network Architecture in Collagen Mechanics. Biophys. J. 2018, 114, 2665-2678.

(47) Shivers, J. L.; Feng, J.; Sharma, A.; MacKintosh, F. C. Normal Stress Anisotropy and Marginal Stability in Athermal Elastic Networks. Soft Matter 2019, 15, 1666-1675.

(48) Wen, Q.; Basu, A.; Janmey, P. A.; Yodh, A. G. Non-Affine Deformations in Polymer Hydrogels. Soft Matter 2012, 8, 8039-8049. 